\title{
On Group Ring Automorphisms
}

\author{
MARTIN HERTWECK ${ }^{1, *}$ and GABRIELE NEBE ${ }^{2}$ \\ ${ }^{1}$ Mathematisches Institut B, Universität Stuttgart, Pfaffenwaldring 57, D-70569 Stuttgart, \\ Germany.email: hertweck@mathematik.uni-stuttgart.de \\ ${ }^{2}$ Universität Ulm, Abteilung Reine Mathematik, D-89069 Ulm, Germany. email: \\ nebe@mathematik.uni-ulm.de
}

\begin{abstract}
For a finite group $G$, the group Outcent $\left(\mathbb{Z}_{p} G\right)$ of outer central automorphisms of $\mathbb{Z}_{p} G$ only depends on the Morita equivalence class of $\mathbb{Z}_{p} G$, which allows reduction to a basic order for its calculation. If the group ring is strongly related to a graduated order, it is often possible to give an explicit description of the basic order (see $[14,13])$. In this paper, we show that Outcent $(B)=1$ for a block $B$ of $\mathbb{Z}_{p} G$ with cyclic defect group. We also prove that Outcent $\left(\mathrm{B}_{0}^{(3)}\left(A_{6}\right)\right)=1$ for the principal block $\mathrm{B}_{0}^{(3)}\left(A_{6}\right)$ of $\mathbb{Z}_{3} A_{6}$; this allows us to verify a conjecture of Zassenhaus for the perfect group of order 1080.
\end{abstract}

Keywords: integral group ring; automorphism; conjecture of Zassenhaus.

Mathematics Subject Classifications (2000): 20C10, 20C05, 16U60.

\section{Introduction}

Automorphisms of group rings $R G$, where $G$ is a finite group and $R$ a $p$ adic ring, have been studied with great success in the past two decades (see $[15-18,9,10])$; the main research was centered round the Zassenhaus conjectures. Observe that not much is lost by restricting attention to normalized automorphisms, i.e., to automorphisms which map $G$ into the group of units of augmentation 1 in $R G$. Recall that a central automorphism of a ring $\Lambda$ is a ring automorphism of $\Lambda$ which fixes the center of $\Lambda$ element-wise. One of the Zassenhaus conjectures in its 'automorphism form' can be stated as follows (see [19, Section 37]):

(ZC) $)_{\text {Aut }}$ Each normalized automorphism of $\mathbb{Z} G$ is the composition of a group automorphism of $G$ (extended to a ring automorphism) and a central automorphism of $\mathbb{Z} G$.

Though not true in general, (ZC) Aut holds for a wide range of groups $G$ (we refer to $[3,4,15,19]$ for a survey on known results).

We fix some notation. Let $R$ be a commutative ring. By $\operatorname{Aut}_{\mathrm{n}}(R G)$ we denote the group of normalized $R$-algebra automorphisms of $R G$. If a ring $\Lambda$ is an $R$-algebra, then $\operatorname{Aut}(\Lambda)$ denotes the group of automorphisms of $\Lambda$ as $R$-algebra, and $\operatorname{Out}(\Lambda)$ the quotient of $\operatorname{Aut}(\Lambda)$ by $\operatorname{Inn}(\Lambda)$, the group of

* The first author was supported by the Deutsche Forschungsgemeinschaft.

(C) 2001 Kluwer Academic Publishers. Printed in the Netherlands. 
inner automorphisms of $\Lambda$. By Autcent $(\Lambda)$ we denote the group of central automorphisms of $\Lambda$, and by Outcent $(\Lambda)$ its image in Out $(\Lambda)$ (changing "A" to "O" will always have this fixed meaning).

Note that the center of $\mathbb{Z} G$ is the $\mathbb{Z}$-span of the class sums, i.e. the sums over the elements of a conjugacy class of $G$. It is well known that a normalized automorphism of $\mathbb{Z} G$ permutes the class sums (see [19, (36.5)]). Thus $\operatorname{Aut}_{\mathrm{n}}(\mathbb{Z} G)$ acts on the character table of $G$, and the conjecture $(\mathrm{ZC})_{\text {Aut }}$ asserts that this action coincides with the action of $\operatorname{Aut}(G)$. We verify this criterion for the perfect group of order 1080, which is a central extension of the alternating group $A_{6}$ by a cyclic group of order 3 , in Section 5. This will follow from general considerations as soon as we have established that Outcent $\left(\mathrm{B}_{0}^{(3)}\left(A_{6}\right)\right)=1$ for the principal block $\mathrm{B}_{0}^{(3)}\left(A_{6}\right)$ of $\mathbb{Z}_{3} A_{6}$; that is done in Section 4. Here we merely remark that the 3-decomposition numbers of $\mathrm{B}_{0}^{(3)}\left(A_{6}\right)$ are 0 or 1 , so $\mathrm{B}_{0}^{(3)}\left(A_{6}\right)$ is strongly related to a graduated order in the sense of [14, Definition II.1], and an explicit description of the basic order which is Morita equivalent to $\mathbf{B}_{0}^{(3)}\left(A_{6}\right)$ has been given in [13]. Furthermore, Outcent $(-)$ is, with regard to $p$-adic group rings, invariant under Morita equivalence. This is shown in Section 1, where we collect some general facts about automorphisms of Morita equivalent rings.

In general, knowledge about the automorphism groups of blocks of group rings over $p$-adic integers should contribute to the understanding of automorphisms and isomorphisms of integral group rings (cf. [17, 18]).

Now let $R$ be a $p$-adic ring, i.e. the integral closure of the $p$-adic integers $\mathbb{Z}_{p}$ in a finite extension field of the $p$-adic field $\mathbb{Q}_{p}$. Let $B$ be a block of $R G$, with defect group $D$, and let $B_{0}$ be the principal block of $R G$, with defect group a Sylow $p$-subgroup $P$ of $G$. We denote by $\operatorname{Aut}_{0}(B)$ the subgroup of all automorphisms of $\operatorname{Aut}(B)$ stabilizing the isomorphism classes of all simple $B$-modules, and by $\operatorname{Aut}_{D}(B)$ the subgroup of all automorphisms of $\operatorname{Aut}(B)$ (respectively of $\operatorname{Aut}_{\mathrm{n}}(B)$, if $B$ is the principal block) stabilizing $D$, where $D$ is identified with its projection on $B$ (we should remark that in [12], the latter notation has an other meaning).

Note that the question whether there is an equality $\operatorname{Out}_{\mathrm{n}}\left(B_{0}\right)=\operatorname{Out}_{P}\left(B_{0}\right)$ is a special case of the "defect group conjugacy problem" raised by Scott ([17, p. 267], [18]); a first positive answer, for $p$-groups $G$, was given by Roggenkamp and Scott in their fundamental paper [16].

Furthermore, we would like to mention that, given $\alpha \in \operatorname{Aut}(B)$, the block $B$ can be viewed as a 'twisted' $(R G, R G)$-bimodule ${ }_{\alpha} B_{1}$ (left action twisted by $\alpha$ ), and if $\alpha \in \operatorname{Aut}_{D}(B)$, the Green correspondent of ${ }_{\alpha} B_{1}$ is the Brauer correspondent $b$, viewed as a 'twisted' bimodule ${ }_{\beta} b_{1}$, where $\beta \in \operatorname{Aut}(b)$ agrees with $\alpha$ on $D$ (see [15, (4.8)]). If $\alpha \in \operatorname{Aut}_{P}\left(B_{0}\right)$ fixes $P$ element-wise, then $\alpha \in \operatorname{Inn}\left(B_{0}\right)$ (see [10, Proposition 4.3]; this is clearly a result for the principal block). Thus $\operatorname{Out}_{P}\left(B_{0}\right)$ can be considered as a subgroup of $\operatorname{Out}(P)$. 
If $G$ is a $p$-constrained group (so in particular, if $G$ is solvable), and $\bar{G}=$ $G / \mathrm{O}_{p^{\prime}}(G)$, then $\operatorname{Out}_{P}\left(B_{0}\right) \cong \operatorname{Out}(\bar{G})\left(\right.$ see [9]) and $\operatorname{Outcent}\left(B_{0}\right) \cong \operatorname{Out}_{\mathrm{c}}(\bar{G})$, the group of outer class preserving automorphisms of $\bar{G}$ (this follows from a theorem due to Roggenkamp and Scott which we call the "F*-Theorem", see $[17,15,10,9])$. However, with respect to the defect group conjugacy problem, Scott pointed out that "In the nonsolvable case there is not yet any empirical evidence one way or another." ([17, p. 268]).

The Green correspondence for automorphisms mentioned above and the results for $p$-constrained groups suggest that the defect group conjugacy problem for the principal block $B_{0}$ is correlated to questions about the structure of Outcent $\left(B_{0}\right)$. For example, let $B_{0}=\mathrm{B}_{0}^{(p)}\left(\mathrm{SL}_{2}\left(p^{f}\right)\right)$ be the principal $p$-block of $\mathrm{SL}_{2}\left(p^{f}\right)$. Then $\operatorname{Out}_{P}\left(B_{0}\right)=\operatorname{Out}\left(B_{0}\right)$ would imply that Outcent $\left(B_{0}\right)=1$ (see Proposition 4.6). Thus it seems worthwhile to calculate some of the groups Outcent $\left(B_{0}\right)$.

It is natural to first lookup the situation when the defect group is cyclic. $\operatorname{Then}_{\operatorname{Out}_{\mathrm{n}}}\left(B_{0}\right)=\operatorname{Out}_{P}\left(B_{0}\right)$ (see [18], [12, Corollary 5.8]). Here, we show that Outcent $(B)$ is trivial for a block $B$ with cyclic defect group, using Plesken's description of the ring theoretical structure of $B$ ([14, Chapter 8$])$. This will be done in Section 3. Section 1 contains some remarks on automorphisms of graduated orders which will used for the proofs.

Finally, we would like to point out that a ' $p$-adic version' of the conjecture of Zassenhaus does not hold for principal $p$-blocks with cyclic defect group. For example, let $B_{0}=\mathrm{B}_{0}^{(11)}\left(M_{11}\right)$ be the principal 11-block of the Mathieu group $M_{11}$. The normalizer $H=\mathrm{N}_{M_{11}}(P)$ of a Sylow 11-subgroup $P$ of $M_{11}$ is the Frobenius group of order 55. Thus induction and restriction induce a stable equivalence of Morita type between $B_{0}$ and $R H$ (in the sense of [12, Definition 3.2], see [12, Proposition 6.1]), and $\operatorname{Out}_{n}\left(B_{0}\right)=\operatorname{Out}_{0}\left(B_{0}\right) \cong C_{2}$, a cyclic group of order 2 by [12, Theorem 5.6]. Thus there are outer automorphisms which cannot be compensated by group automorphisms since $\operatorname{Out}\left(M_{11}\right)=1$. (A similar remark holds for various other sporadic groups.)

\section{Automorphisms of Morita equivalent rings}

Let $R$ be a commutative ring and suppose that $\Lambda$ is an $R$-algebra. For an idempotent $f$ of $\Lambda$, we denote by $\operatorname{Aut}(\Lambda, f)$ the subgroup of all automorphisms of $\operatorname{Aut}(\Lambda)$ fixing $f$, with image $\operatorname{Out}(\Lambda, f)$ in $\operatorname{Out}(\Lambda)$. Similarly, we define Outcent $(\Lambda, f)$.

Let $f$ be an idempotent of $\Lambda$ such that $\Lambda f \Lambda=\Lambda$, where $\Lambda f \Lambda$ denotes the ideal generated by $f$, and set $\Gamma=f \Lambda f$. Then the $(\Lambda, \Gamma)$-bimodule $\Lambda f$ and the $(\Gamma, \Lambda)$-bimodule $f \Lambda$ are bimodules inducing a Morita equivalence between the $R$-algebras $\Lambda$ and $\Gamma$. 
Clearly, restriction induces a homomorphism $\operatorname{Aut}(\Lambda, f) \rightarrow \operatorname{Aut}(\Gamma)$. If $\alpha$ is an inner automorphism of $\Lambda$, say conjugation with $u \in \Lambda^{\times}$, which fixes $f$, then $f$ commutes with $u$ and $\alpha(\gamma)=u^{-1} \gamma u=\left(f u^{-1} f\right) \gamma(f u f)$ for all $\gamma \in \Gamma$, so $\alpha$ induces an inner automorphism of $\Gamma$, given by conjugation with the unit $f u f \in \Gamma^{\times}$. Thus, the homomorphism $\operatorname{Aut}(\Lambda, f) \rightarrow \operatorname{Aut}(\Gamma)$ gives rise to a homomorphism $1: \operatorname{Out}(\Lambda, f) \rightarrow \operatorname{Out}(\Gamma)$.

PROPOSITION 1.1. The homomorphism $1: \operatorname{Out}(\Lambda, f) \rightarrow \operatorname{Out}(\Gamma)$ above is injective, and maps $\operatorname{Outcent}(\Lambda, f)$ into $\operatorname{Outcent}(\Gamma)$.

Proof. Let $\alpha \in \operatorname{Aut}(\Lambda)$ with $\alpha(f)=f$, and assume that $\alpha$ induces an inner automorphism of $\Gamma$, say conjugation with $v \in \Gamma^{\times}$. Modifying $\alpha$ by conjugation with the unit $v+(1-f)$ of $\Lambda$, we may assume that $\left.\alpha\right|_{\Gamma}=\left.\mathrm{id}\right|_{\Gamma}$. It follows that $f \Lambda \stackrel{\alpha}{\rightarrow} f \Lambda$ is an automorphism of $\Gamma$-left modules. Since $\operatorname{End}_{\Gamma}(f \Lambda)^{\mathrm{op}}=$ $\Lambda$, there is $u \in \Lambda^{\times}$with $\alpha(f \lambda)=f \lambda u$ for all $\lambda \in \Lambda$. Similarly $\alpha(\lambda f)=u^{\prime} \lambda f$ for some $u^{\prime} \in \Lambda^{\times}$and all $\lambda \in \Lambda$. Therefore $\alpha\left(\lambda^{\prime} f \lambda\right)=u^{\prime} \lambda^{\prime} f \lambda u$ for all $\lambda, \lambda^{\prime} \in \Lambda$. Since $\Lambda=\Lambda f \otimes_{\Gamma} f \Lambda$, it follows that $\alpha(\lambda)=u^{\prime} \lambda u$ for all $\lambda \in \Lambda$. Now $\alpha(1)=1$ implies that $u^{\prime}=u^{-1}$, so $\alpha$ is an inner automorphism of $\Lambda$, and we have seen that $l$ is injective.

Recall that there is an isomorphism $\phi: Z(\Lambda) \rightarrow Z(\Gamma)$ such that for each $c \in$ $\mathrm{Z}(\Lambda)$, the element $\phi(c)$ is the unique element in $\mathrm{Z}(\Gamma)$ such that $c \cdot m=m \cdot \phi(c)$ for all $m \in \Lambda f$ (see [6, (55.7)]). In particular, $c f=\phi(c)$ for all $c \in \mathrm{Z}(\Lambda)$, and it follows that a central automorphism $\alpha$ of $\Lambda$ with $\alpha(f)=f$ induces a central automorphism $\left.\alpha\right|_{\Gamma}$ of $\Gamma$. This shows that 1 restricts to an inclusion Outcent $(\Lambda, f) \hookrightarrow \operatorname{Outcent}(\Gamma)$.

We now consider the particular case that $R$ is a complete discrete valuation ring of characteristic 0 , with field of fractions $K$, and that $\Lambda$ is an $R$-order in a separable $K$-algebra $A$. Let $e_{1}, \ldots, e_{h} \in \Lambda$ be orthogonal primitive idempotents such that $e_{1} \Lambda, \ldots, e_{h} \Lambda$ represent the isomorphism types of the projective indecomposable $\Lambda$-right modules, and set $f=e_{1}+\ldots+e_{h}$.

COROLLARY 1.2. With notation as above, there is an injective homomorphism $\operatorname{Out}(\Lambda) \rightarrow \operatorname{Out}(f \Lambda f)$ which maps Outcent $(\Lambda)$ into Outcent $(f \Lambda f)$.

Proof. It is well known that $\Lambda$ is Morita equivalent to the $R$-order $\Gamma=f \Lambda f$ (as general reference we give [21]). Moreover, Aut $(\Lambda, f)$ covers the outer automorphism group $\operatorname{Out}(\Lambda)$, that is, $\operatorname{Aut}(\Lambda)=\operatorname{Aut}(\Lambda, f) \cdot \operatorname{Inn}(\Lambda)$, since the set $\left\{e_{1}, \ldots, e_{h}\right\}$ is unique up to conjugacy with units of $\Lambda$. Thus, the inclusion $\operatorname{Out}(\Lambda, f) \rightarrow \operatorname{Out}(\Gamma)$ gives rise to injective homomorphisms $\operatorname{Out}(\Lambda) \rightarrow$ $\operatorname{Out}(\Gamma)$ and Outcent $(\Lambda) \rightarrow \operatorname{Outcent}(\Gamma)$.

We also remark that each central automorphism of $\Lambda$ is given by conjugation with a unit of $A$, by the theorems of Skolem-Noether and NoetherDeuring. Thus if $\mathrm{N}(\Lambda)=\left\{x \in A^{\times} \mid x^{-1} \Lambda x=\Lambda\right\}$, the normalizer of $\Lambda$ in the 
units of $A$, and $C$ denotes the center of $\Lambda$ (so that $K C$ is the center of $A$ ), then Outcent $(\Lambda)=\mathrm{N}(\Lambda) / \Lambda^{\times}(K C)^{\times}$.

\subsection{GROUP RINGS}

We now specialize to the case of group rings. Let $G$ be a finite group, and let $R$ be a complete discrete valuation ring of characteristic 0 , with field of fractions $K$ and residue field $k$ of characteristic $p$.

We first record a lemma which is derived from Brauer's proof on the number of simple $k G$-modules ( $k$ sufficiently large). We use the following notation.

NOTATION 1.3. The class sum of $g \in G$, i.e. the sum of the conjugates of $g$ in $G$, is denoted by $\mathcal{C}_{g}$, and the class length $\left|\mathcal{C}_{g}\right|$ of $g$ is the number of conjugates of $g$ in $G$.

LEMMA 1.4. An automorphism of $R G$ which fixes the class sums of $p^{\prime}$ elements of $G$ maps any idempotent of $R G$ to some conjugate of it.

Proof. Let $\alpha$ be an automorphism of $R G$ which fixes the class sums of $p^{\prime}$ elements of $G$, and let $e$ be an idempotent of $R G$. Note that $e$ and $\alpha(e)$ are conjugate by a unit of $R G$ if and only if $R G e \cong R G \alpha(e)$ as left $R G$-modules, and that this happens if $S G e \cong S G \alpha(e)$ as left $S G$-modules for some extension $S$ of $R$ (see [6, (30.25)]). Thus we can assume that $k$ is a splitting field for $k G$.

Let $V=[R G, R G]$ be the subspace of $R G$ spanned by all elements $x y-y x$ $(x, y \in R G)$, and let $g$ be a $p^{\prime}$-element of $G$. As $\mathcal{C}_{g}+V=\left|\mathcal{C}_{g}\right| g+V$,

$$
\left|\mathcal{C}_{g}\right|(\alpha(g)-g)=\alpha\left(\left|\mathcal{C}_{g}\right| g\right)-\left|\mathcal{C}_{g}\right| g \equiv \alpha\left(\mathcal{C}_{g}\right)-\mathcal{C}_{g}=0 \quad \bmod V .
$$

Since $R G / V$ is $R$-torsionfree (see [6, (32.3)]), it follows that $\alpha(g)+V=g+V$.

Set $T=[k G, k G]$ and $S=T+\operatorname{rad}(k G)$. Let $x_{1}, \ldots, x_{s}$ be representatives of the $s$ conjugacy classes of $p^{\prime}$-elements of $G$. Then $x_{1}+S, \ldots, x_{s}+S$ form a basis of $k G / S$ (see [1, p. 18]), so $\alpha$ induces the identity on $k G / S$. Note that $S / \operatorname{rad}(k G)$ is just $[k G / \operatorname{rad}(k G), k G / \operatorname{rad}(k G)]$, i.e., consists of 'matrices' of trace zero. Thus $\alpha$ maps each primitive idempotent of $k G / \operatorname{rad}(k G)$ to a conjugate of it (see $[21,(1.15)]$ ), and the lemma follows from [21, (3.1), (4.16)].

In particular, a central automorphism $\alpha$ of $R G$ maps any idempotent $e$ of $R G$ to a conjugate of it. This can also be seen in the following way. By the Skolem-Noether theorem, the idempotents $e$ and $\alpha(e)$ are conjugate in $K G$, so the $K G$-modules $K G e$ and $K G \alpha(e)$ are isomorphic. Since projective modules are determined by their characters (see [6, (18.16)]), it follows that $R G e \cong R G \alpha(e)$ as $R G$-modules. Therefore $e$ and $\alpha(e)$ are conjugate by a unit of $R G$. 
In other words, every central automorphism of a block of a group ring fixes all irreducible Brauer characters of this block (this follows also from the surjectivity of the decomposition map). Of course this is not necessarily true for epimorphic images of the block (i.e., for the projections onto blocks of $K G)$. Thus we define for an $R$-order $\Gamma$ the subgroup

$$
\begin{gathered}
\text { Autcent }_{0}(\Gamma):=\{\alpha \in \operatorname{Autcent}(\Gamma) \mid \alpha \text { stabilizes the isomorphism classes } \\
\text { of all simple } \Gamma \text {-modules }\}
\end{gathered}
$$

of Autcent $(\Gamma)$ in which we will be interested in. We record explicitly:

COROLLARY 1.5. Let $e_{1}, \ldots, e_{h}$ be orthogonal idempotents which lift the central primitive idempotents of $R G / \operatorname{rad}(R G)$ and sum up to 1 . Then every class in $\operatorname{Outcent}(R G)$ is represented by an automorphism $\alpha$ with $\alpha\left(e_{i}\right)=e_{i}$ for $1 \leq i \leq h$, and Outcent $(R G)=\operatorname{Outcent}_{0}(R G)$.

Of course, the corollary can be stated analogously for blocks of group rings, and the following discussion will show that it also holds for orders that are Morita equivalent to blocks of group rings.

To this aim, recall from $[6,(55.5),(55.6)]$ the definition of the Picard groups $\operatorname{Pic}_{R}(\Lambda)$ and Picent $(\Lambda)$. We will need the following proposition, which combines [6, Exercises 55.7, 55.8] with [6, (55.9)].

PROPOSITION 1.6. Let $\Lambda$ and $\Gamma$ be Morita equivalent $R$-algebras, and set $\bar{\Lambda}=\Lambda / \operatorname{rad}(\Lambda), \bar{\Gamma}=\Gamma / \operatorname{rad}(\Gamma)$. Assume that $\bar{\Lambda}$ is semisimple artinian. Then there is a commutative diagram

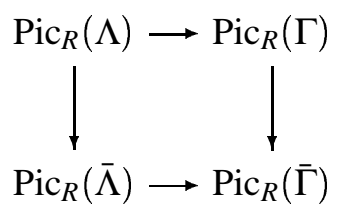

where the horizontal maps are isomorphisms. The isomorphism $\operatorname{Pic}_{R}(\Lambda) \rightarrow$ $\operatorname{Pic}_{R}(\Gamma)$ restricts to an isomorphism Picent $(\Lambda) \rightarrow \operatorname{Picent}(\Gamma)$.

The group Outcent $0(\Lambda)$, viewed as a subgroup of $\operatorname{Pic}_{R}(\Lambda)$, lies in the kernel of the homomorphism $\operatorname{Pic}_{R}(\Lambda) \rightarrow \operatorname{Pic}_{R}(\bar{\Lambda})$.

Proof. Let $M$ be a $(\Lambda, \Gamma)$-bimodule which gives a Morita equivalence over $R$ between $\Lambda$ and $\Gamma$. Put $\bar{M}=M / \operatorname{rad}(\Lambda) M$, a $(\bar{\Lambda}, \Gamma)$-bimodule, and set $E=\operatorname{End}_{\bar{\Lambda}}(\bar{M})$. Then $E$ is semisimple artinian, and may be viewed as a ring of right operators on $\bar{M}$. The map sending $\gamma$ to right multiplication with $\gamma$ on $\bar{M}$ gives a ring homomorphism $\varphi: \Gamma \rightarrow E$, and $\varphi$ is surjective since every $\bar{\Lambda}$-homomorphism of $\bar{M}$ lifts to a $\Lambda$-homomorphism of $M$ ( $M$ being $\Lambda$-projective), and hence is right multiplication with some $\gamma \in \Gamma$ since $\Gamma=$ $\operatorname{End}_{\Lambda}(M)$. It follows that $\varphi(\operatorname{rad}(\Gamma)) \subseteq \operatorname{rad}(E)=0$ (see [6, (5.6)]), so $\bar{M}$ is a $\bar{\Gamma}$-module. 
That $\bar{M}$ is a finitely generated projective $\bar{\Lambda}$-module and a generator of the category of $\bar{\Lambda}$-modules follows easily from the corresponding properties of $M$. Hence $\bar{\Lambda}$ and $\Gamma / \operatorname{ker}(\varphi)$ are Morita equivalent. Since $\Lambda$ and $\Gamma$ have the same number of simple modules, it follows that $\operatorname{ker}(\varphi)=\operatorname{rad}(\Gamma)$. Note that $\bar{M}$ gives a Morita equivalence over $R$ between $\bar{\Lambda}$ and $\bar{\Gamma}$.

Assume for a moment that $\Lambda=\Gamma$. Then $(M)$, the isomorphism class of $M$, is a typical element of $\operatorname{Pic}_{R}(\Lambda)$, and we have shown that $(\bar{M})$ is an element of $\operatorname{Pic}_{R}(\bar{\Lambda})$. Thus we obtain a homomorphism $\operatorname{Pic}_{R}(\Lambda) \rightarrow \operatorname{Pic}_{R}(\bar{\Lambda})$, and likewise a homomorphism $\operatorname{Pic}_{R}(\Gamma) \rightarrow \operatorname{Pic}_{R}(\bar{\Gamma})$.

There are isomorphisms $\operatorname{Pic}_{R}(\Lambda) \rightarrow \operatorname{Pic}_{R}(\Gamma),(X) \mapsto\left(M^{-1} \otimes_{\Lambda} X \otimes_{\Lambda} M\right)$ and $\operatorname{Pic}_{R}(\bar{\Lambda}) \rightarrow \operatorname{Pic}_{R}(\bar{\Gamma}),(Y) \mapsto\left(\bar{M}^{-1} \otimes_{\bar{\Lambda}} Y \otimes_{\bar{\Lambda}} \bar{M}\right)$ (see [6, (55.9)]). It is easily checked that the given maps give rise to a commutative diagram, as shown above.

The map $\operatorname{Pic}_{R}(\Lambda) \rightarrow \operatorname{Pic}_{R}(\Gamma)$ restricts to a map Picent $(\Lambda) \rightarrow \operatorname{Picent}(\Gamma)$ (see $[6,(55.9)])$. By definition, Outcent ${ }_{0}(\Lambda)$ maps under the homomorphism $\operatorname{Pic}_{R}(\Lambda) \rightarrow \operatorname{Pic}_{R}(\bar{\Lambda})$ into Picent $(\bar{\Lambda})$, and this group is trivial by [6, (55.15)].

A basic result in the calculation of Picard groups of group rings is the following (see $[6, \S 55]$ ):

$$
\operatorname{Picent}(R G) \cong \operatorname{Outcent}(R G) \cong \mathrm{N}(R G) /(R G)^{\times}(K C)^{\times} .
$$

As Picard groups are invariant under Morita equivalence, this result allows the following reduction in the calculation of Outcent $(R G)$. We follow the discussion given in [6] subsequent to the proof of (55.23).

PROPOSITION 1.7. Let $\Gamma$ be an R-order which is Morita equivalent to RG. Then Outcent $(R G) \cong$ Outcent $_{0}(\Gamma)$.

Proof. Set $\Lambda=R G$. By $[6, \S 55]$, there are isomorphisms

$$
\operatorname{Outcent}(\Lambda) \cong \operatorname{Picent}(\Lambda) \cong \operatorname{Picent}(\Gamma) \text {. }
$$

We first show that Picent $(\Gamma) \cong \operatorname{Outcent}(\Gamma)$. Let $M$ be a $(\Lambda, \Gamma)$-bimodule which gives a Morita equivalence between $\Lambda$ and $\Gamma$. Note that $K \Gamma$ is a finite dimensional semisimple $K$-algebra. Let $(X) \in \operatorname{Picent}(\Gamma)$. By [6, (55.15), (55.17)], $K X \cong K \Gamma$ as bimodules. Since $X$ is a projective left $\Gamma$-module, and the functor $M \otimes_{\Gamma}$ - provides a Morita equivalence between the categories of $\Gamma$-modules and $\Lambda$-modules, $M \otimes_{\Gamma} X$ is a projective left $\Lambda$-module. There is an embedding of left $K \Lambda$-modules $K \otimes_{R}\left(M \otimes_{\Gamma} X\right) \hookrightarrow K \otimes_{R} M \otimes_{\Gamma} K X=K \otimes_{R} M$, which is clearly surjective. By Swan's theorem [6, (32.1)], it follows that $M \otimes_{\Gamma} X \cong M$ as $\Lambda$-modules. Therefore $X \cong \Gamma \otimes_{\Gamma} X \cong M \otimes_{\Lambda} M \otimes_{\Gamma} X \cong M \otimes_{\Lambda} M \cong \Gamma$ as left $\Gamma$-modules, and it follows that Picent $(\Gamma) \cong \operatorname{Outcent}(\Gamma)$.

Thus Outcent $(\Lambda) \cong \operatorname{Outcent}(\Gamma)$. Note that Outcent $(\Lambda)=\operatorname{Outcent}_{0}(\Lambda)$ by Corollary 1.5, so it follows from Proposition 1.6 that Outcent $(\Gamma)$ lies in the 
kernel of the homomorphism $\operatorname{Pic}_{R}(\Gamma) \rightarrow \operatorname{Pic}_{R}(\bar{\Gamma})$, where $\bar{\Gamma}=\Gamma / \operatorname{rad}(\Gamma)$, and therefore Outcent $(\Gamma)=$ Outcent $_{0}(\Gamma)$. This completes the proof.

Let $f$ be an idempotent of $R G$ such that $R G$ is Morita equivalent to the $R$-order $f R G f$. We remark that Proposition 1.1 and Lemma 1.4 already show that there is a natural inclusion $\operatorname{Outcent}(R G) \hookrightarrow \operatorname{Outcent}(f R G f)$, which can be shown to be surjective by constructing explicitly preimages:

Let $1=e_{1}+\ldots+e_{m}+e_{m+1}+\ldots+e_{n}$ be a primitive decomposition of $1 \epsilon$ $R G$ such that $f=e_{m+1}+\ldots+e_{n}$ is a primitive decomposition of $f \in f R G f$ (see [21, (4.12)]). There is a map $\sigma:\{1, \ldots, m\} \rightarrow\{m+1, \ldots, n\}$ and units $u_{i} \in R G$ such that $e_{i}=u_{i}^{-1} e_{\sigma(i)} u_{i}$ for $1 \leq i \leq m$ (see [21, (9.9)]).

Fix a coset in Outcent $(f R G f)$, with representative $\beta$. As mentioned above, there is $v \in(f K G f)^{\times}$such that $\beta(x)=v^{-1} x v$ for all $x \in f R G f$. Fix some $m+1 \leq i \leq n$. Since $e_{i}$ and $\beta\left(e_{i}\right)$ are conjugate in the units of $K G$, the $K G$ modules $K G e_{i}$ and $K G \beta\left(e_{i}\right)$ are isomorphic. It follows from [6, (18.16)]) that $R G e_{i} \cong R G \beta\left(e_{i}\right)$ as $R G$-modules, and therefore $w_{i}^{-1} e_{i} w_{i}=\beta\left(e_{i}\right)$ for some $w_{i} \in$ $(R G)^{\times}$. Then $w=\sum_{i=m+1}^{n} e_{i} w_{i} \beta\left(e_{i}\right)$ is a unit in $f R G f$ with $w^{-1} e_{i} w=\beta\left(e_{i}\right)$ for all $m+1 \leq i \leq n$. Thus we can assume that $\beta$ fixes all $e_{i}$. Then it is easily checked that

$$
u=v+\sum_{i=1}^{m} e_{i} u_{i}^{-1} v u_{i} e_{i} \in(K G)^{\times}, \quad \text { with } \quad u^{-1}=v^{-1}+\sum_{i=1}^{m} e_{i} u_{i}^{-1} v^{-1} u_{i} e_{i} .
$$

By construction, $u^{-1} R G u=R G$, so conjugation with $u$ induces a central automorphism $\alpha$ of $R G$. Furthermore, $\alpha(f)=f$ and $\left.\alpha\right|_{f R G f}=\beta$. Thus the coset of $\alpha$ in $\operatorname{Outcent}(R G)$ is a preimage of the coset of $\beta$.

\section{Reduction and extension of scalars}

Let $R$ be a $p$-adic ring, fix $\pi \in R$ with $\operatorname{rad}(R)=\pi R$ and let $e$ be the ramification index of $K / \mathbb{Q}_{p}$, where $K$ is the quotient field of $R$. Thus $p R=\pi^{e} R$. The following theorem has been proved in $[10,3.13]$.

THEOREM 1.8. Let $t \in \mathbb{N}$ with $t>e /(p-1)$. Then any automorphism $\alpha$ of $R G$ which induces the identity on $\left(R / \pi^{t} R\right) G$ is an inner automorphism of $R G$.

Let $f$ be an idempotent of $R G$ such that $R G$ is Morita equivalent to the $R$-order $f R G f$, and set $\Lambda=R G, \Gamma=f R G f$. Fix some $t \in \mathbb{N}$, and let bars denote reduction mod $\pi^{t}$, i.e. write $\bar{\Lambda}=R / \pi^{t} R \otimes_{R} \Lambda=\left(R / \pi^{t} R\right) G$. Clearly, the bimodules $\bar{f} \bar{\Lambda}$ and $\bar{\Lambda} \bar{f}$ realize a Morita equivalence between the $R / \pi^{t} R$ algebras $\bar{\Lambda}$ and $\bar{\Gamma}=\bar{f} \bar{\Lambda} \bar{f}$.

The homomorphism $\operatorname{Aut}(\Lambda, f) \rightarrow \operatorname{Aut}(\bar{\Lambda})$ which sends an $\alpha$ to id $\otimes \alpha$ induces a homomorphism $\phi_{t}: \operatorname{Out}(\Lambda, f) \rightarrow \operatorname{Out}(\bar{\Lambda})$. Similarly, we get a homomorphism $\operatorname{Out}(\Gamma) \rightarrow \operatorname{Out}(\bar{\Gamma})$, for simplicity also denoted by $\phi_{t}$. Thus we 
have a commutative diagram

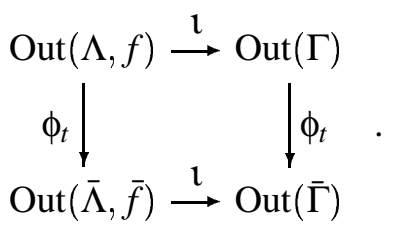

By Proposition 1.1, the horizontal maps are injective. Since the map $\Lambda \rightarrow \bar{\Lambda}$ is surjective on units, Theorem 1.8 has the following consequence.

THEOREM 1.9. The composite homomorphism $1 \circ \phi_{t}: \operatorname{Out}(\Lambda, f) \rightarrow \operatorname{Out}(\bar{\Gamma})$ is injective provided that $t>e /(p-1)$.

Finally, we note that if $S$ is a discrete valuation ring containing $R$, then the natural map $\operatorname{Out}(R G) \rightarrow \operatorname{Out}(S G)$ is injective. This follows from the interpretation of an automorphism as an invertible bimodule and a result due to Reiner and Zassenhaus (see [6, $\$ 35$ exercise 13, (30.25)]).

\section{Graduated orders}

Let $G$ and $(K, R, k)$ as before. We now assume that $K$ is big enough such that $k$ is a splitting field for $k G$, that $K G \varepsilon_{t} \cong K_{t}^{d_{t} \times d_{t}}$ for some totally ramified field extension $K_{t}$ of $K$ and that the center $\mathrm{Z}\left(R G \varepsilon_{t}\right)$ is the maximal order $R_{t}$ in $K_{t}$. We also assume that the decomposition numbers of $R G$ are all 0 or 1, which is the only really restrictive assumption here. Let $e_{1}, \ldots, e_{h}$ be orthogonal idempotents which lift the central primitive idempotents of $R G / \operatorname{rad}(R G)$ and sum up to 1 , and let $\varepsilon_{1}, \ldots, \varepsilon_{s}$ be the central primitive idempotents of $K G$.

Under these assumptions one can show that $e_{i} R G e_{i}$ is a maximal order in $e_{i} K G e_{i}$ and that $R G \varepsilon_{t}$ is conjugate to a graduated order in the simple algebra $K G \varepsilon_{t}$ (see [14]). If $r_{t}=\left\{1 \leq i \leq h \mid \varepsilon_{t} e_{i} \neq 0\right\}$ is the index set specifying the set of $p$-modular constituents of the character belonging to $\varepsilon_{t}(1 \leq t \leq s)$, and $n_{1}^{(t)}, \ldots, n_{l_{t}}^{(t)}$ are the $k$-dimensions of the respective simple modules, then this means that there is a matrix $M^{(t)}=\left(m_{i j}^{(t)}\right) \in \mathbb{Z}_{\geq 0}^{l_{t} \times l_{t}}$ such that $R G \varepsilon_{t}$ is conjugate to

$$
\Lambda\left(R_{t}, n_{1}^{(t)}, \ldots, n_{l_{t}}^{(t)}, M^{(t)}\right):=\left\{\left(X_{i j}\right)_{i, j=1}^{l_{t}} \mid X_{i j} \in \pi_{t}^{m_{i j}^{(t)}} R_{t}^{n_{i}^{(t)} \times n_{j}^{(t)}}\right\}
$$

where $\pi_{t}$ is a prime element in $R_{t}$. The matrices $M^{(t)}$ are called exponent matrices. Together with $R_{t}$ they describe the order $\bigoplus_{t=1}^{s} R G \varepsilon_{t}$ up to Morita equivalence, where passing to the Morita equivalent basic order simply means that all the $n_{i}^{(t)}$ become 1 . 
For a graduated order $\Gamma=\Lambda\left(R, n_{1}, \ldots, n_{l}, M\right)$ of the form $(*)$, and $1 \leq i \leq l$, let $E_{i}$ be the idempotent diagonal matrix in $\Gamma$ with $(i, i)$-th block entry the identity matrix, and all other entries equal to 0 . We note:

LEMMA 2.1. Let $\Gamma=\Lambda\left(R, n_{1}, \ldots, n_{l}, M\right)$ be a graduated order. Then

$$
\text { Outcent }_{0}(\Gamma)=1 \text {. }
$$

More precisely, each $\alpha \in \operatorname{Autcent}(\Gamma)$ which fixes the idempotents $E_{i}$ defined above is conjugation with a block diagonal matrix $\operatorname{diag}\left(A_{j} \mid 1 \leq j \leq l\right) \in \Gamma^{\times}$ with $A_{j} \in\left(R^{n_{j} \times n_{j}}\right)^{\times}$.

Proof. Let $\alpha \in$ Autcent $(\Gamma)$ with $\alpha\left(E_{i}\right)=E_{i}$ for all $1 \leq i \leq l$. By the SkolemNoether theorem, $\alpha$ is conjugation with a matrix $A \in(K \Gamma)^{\times}$. Since $A$ commutes with all idempotents $E_{i}$, it is a block diagonal matrix $\operatorname{diag}\left(A_{j} \mid 1 \leq\right.$ $j \leq l)$ with $A_{j} \in\left(K^{n_{j} \times n_{j}}\right)^{\times}$. Conjugation with $A_{j}$ maps $e_{j} \Gamma e_{j}$, when identified with $R^{n_{j} \times n_{j}}$, into itself, so $A_{j}=\pi^{v_{j}} B_{j}$ with $B_{j} \in\left(R^{n_{j} \times n_{j}}\right)^{\times}$(see [6, (55.23)]). We may assume that $v_{1}=0$. Then conjugation with $A$ maps $\Gamma$ onto $\Lambda\left(R, n_{1}, \ldots, n_{l}, N\right)$ with $n_{i j}=m_{i j}-v_{i}+v_{j}$. Since $A$ preserves $\Gamma$, it follows that $v_{j}=0$ for all $1 \leq j \leq l$, i.e., $A_{j}=B_{j} \in\left(R^{n_{j} \times n_{j}}\right)^{\times}$, and $\alpha$ is, as claimed, conjugation with a block diagonal matrix in $\Gamma^{\times}$.

To prove that Outcent $_{0}(\Gamma)=1$, we may assume that the idempotents $E_{i}$ are lifts of the central primitive idempotents of $\Gamma / \operatorname{rad}(\Gamma)$ (see $[14$, II.3, 4]). But then each coset in $\operatorname{Outcent}_{0}(\Gamma)$ has a representative $\alpha$ of the above form, and the lemma is proved.

Choose an idempotent $f \in R G$ such that $\Lambda=f R G f$ is the Morita equivalent basic order of $R G$, and $f_{i}:=f e_{i}=e_{i} f \in \Lambda(1 \leq i \leq h)$. Then the $f_{i}$ are lifts of the central primitive idempotents of $\Lambda / \operatorname{rad}(\bar{\Lambda})$. We assume (without lost of generality) that for all $i$ and $t$ the element $f_{i} \varepsilon_{t}$ of $\Lambda \varepsilon_{t}$ (identified with a graduated order as above) is a diagonal matrix with at most one entry $\neq 0$ on the diagonal.

COROLLARY 2.2. Let $\Lambda=f R G f$ be as above, and set $\Gamma=\bigoplus_{t=1}^{s} \Lambda \varepsilon_{t}$. If $\alpha \in \operatorname{Autcent}(\Lambda)$ with $\alpha\left(f_{i}\right)=f_{i}$ for $i=1, \ldots, h$, then there is

$$
A=\sum_{t=1}^{s} \operatorname{diag}\left(a_{j}^{(t)} \mid j \in r_{t}\right) \in \Gamma^{\times}
$$

with $a_{j}^{(t)} \in R_{t}^{\times}$such that $\alpha$ is given by conjugation with $A$.

Thus if one investigates Outcent $(\Lambda)$, one should know which of the diagonal matrices $A$ above are in $\Lambda^{\times}$. There are some obvious elements, namely $\sum_{j=1}^{h} a_{j} f_{j} \in \Lambda^{\times}$with $a_{j} \in R^{\times}$(i.e., the $a_{j}^{(t)}$ are independent of $t$ ).

For $1 \leq i \leq h$, let $c_{i}=\left\{1 \leq t \leq s \mid f_{i} \varepsilon_{t} \neq 0\right\}$ be the index set specifying the set of all ordinary irreducible characters which have the irreducible modular 
character belonging to $f_{i}$ as a constituent. One may view the $\left(f_{i} \Lambda f_{i}, f_{j} \Lambda f_{j}\right)$ bimodule $f_{i} \Lambda f_{j}$ as being embedded in $\bigoplus_{t \in c_{i} \cap c_{j}} f_{i} \Lambda f_{j} \varepsilon_{t}$. Then $A$ as in Corollary 2.2 acts on $f_{i} \Lambda f_{j}$ by multiplication with $a_{i}^{(t)-1} a_{j}^{(t)}$ on the component $t \in$ $c_{i} \cap c_{j}$. In particular, if $f_{i} \Lambda f_{j}$ is indecomposable as $\left(f_{i} \Lambda f_{i}, f_{j} \Lambda f_{j}\right)$-bimodule (which is not always, but very often the case), then there is $\alpha_{i j} \in k$ such that $a_{i}^{(t)-1} a_{j}^{(t)}+\pi_{t} R_{t}=\alpha_{i j}$ for all $t \in c_{i} \cap c_{j}$, thus giving additional information on $A$ (this fact will be used in the proof of Theorem 4.1).

In the next section, we will need a simple consequence of Lemma 2.1. Let $\Lambda$ be an $R$-order such that $\Lambda \varepsilon_{t}$ is a graduated order of the form $(*)$ for all central primitive idempotents $\varepsilon_{1}, \ldots, \varepsilon_{s}$ of $K \Lambda$. As above, let $e_{1}, \ldots, e_{h}$ be orthogonal idempotents which lift the central primitive idempotents of $\Lambda / \operatorname{rad}(\Lambda)$ and sum up to 1 , and let $r_{t}=\left\{1 \leq i \leq h \mid \varepsilon_{t} e_{i} \neq 0\right\}$. Assume that $\left|r_{t_{1}} \cap r_{t_{2}}\right| \in\{0,1\}$ for all $1 \leq t_{1}, t_{2} \leq s$. Let $\mathcal{G}$ be the graph whose vertices are indexed by the $\varepsilon_{t}$, with two vertices connected if the corresponding $r_{t}$ have non-empty intersection.

COROLLARY 2.3. If $\mathcal{G}$ is a tree, then $\operatorname{Outcent}_{0}(\Lambda)=1$.

Proof. The proof is by induction on the number $s$ of blocks of $K \Lambda$. In consideration of Lemma 2.1, we can assume that $s>1$. Furthermore, we can assume that $\Lambda$ is indecomposable and basic. Arrange the $\varepsilon_{t}$ so that $\varepsilon_{s}$ corresponds to a leaf of the tree $\mathcal{G}$, and let $\Lambda_{1}=\Lambda\left(\varepsilon_{1}+\ldots+\varepsilon_{s-1}\right)$. The graduated order $\Lambda \varepsilon_{s}$ is contained in a matrix ring over a discrete valuation ring $S$. For some quotient $\bar{S}$ there are epimorphisms $\mu: \Lambda_{1} \rightarrow \bar{S}$ and $\nu: \Lambda \varepsilon_{s} \rightarrow \bar{S}$ such that $\Lambda=\left\{(x, y) \in \Lambda_{1} \oplus \Lambda \varepsilon_{s} \mid \mu(x)=v(y)\right\}$. By induction hypothesis and Lemma 2.1, Outcent ${ }_{0}\left(\Lambda_{1}\right)=1$ and Outcent $0\left(\Lambda \varepsilon_{s}\right)=1$. Since units of $\bar{S}$ can be lifted to central units of $\Lambda \varepsilon_{s}$ (i.e., to multiples of the identity matrix), it follows that $\operatorname{Outcent}_{0}(\Lambda)=1$.

\section{Blocks with cyclic defect groups}

Let $B$ be a block of a group ring $\mathbb{Z}_{p} G$ with cyclic defect group of order $p^{a}$. Let $K$ be the minimal unramified extension of $\mathbb{Q}_{p}$ and $R$ be the ring of integers in $K$ such that the residue class field $R / p R$ is a splitting field for $B / p B$. Let $\tilde{\Lambda}$ be a direct summand of the $R$-order $R \otimes_{\mathbb{Z}_{p}} B$ and $\Lambda$ be the basic order Morita equivalent to $\tilde{\Lambda}$. We will show that $\operatorname{Outcent}_{0}(\Lambda)=1$, which, by the results of Section 1, immediately implies that:

THEOREM 3.1. If B is a block of a group ring $\mathbb{Z}_{p} G$ with cyclic defect group, then $\operatorname{Outcent}(B)=1$.

To fix the notation we repeat the description of $\Lambda$ given in [14, Chapter VIII]: 
There is a divisor $e$ of $p-1$ such that $\tilde{\Lambda}$ contains (over a splitting field) $\frac{p^{a}-1}{e}+e$ irreducible Frobenius characters and $e$ irreducible Brauer characters.

$K \Lambda$ has only $a+e$ central primitive idempotents $\varepsilon_{1}, \ldots, \varepsilon_{a}, \varepsilon_{a+1}, \ldots, \varepsilon_{a+e}$. They can be ordered such that $\varepsilon_{1}, \ldots, \varepsilon_{a}$ belong to the exceptional vertex

$$
\Gamma_{a}:=\left(\varepsilon_{1}+\ldots+\varepsilon_{a}\right) \Lambda,
$$

and such that the center of $K \Lambda \varepsilon_{t}$ is a completely ramified extension $K_{t}$ of $K$ of degree $\left[K_{t}: K\right]=\frac{p^{t}-p^{t-1}}{e}(1 \leq t \leq a)$. Let $R_{t}$ be the ring of integers in $K_{t}$ with prime element $\pi_{t}$. Let $1=e_{1}+\ldots+e_{e}$ be an orthogonal decomposition which lifts a decomposition of 1 in $\Lambda / \operatorname{rad}(\Lambda)$ into central primitive idempotents.

Again, set $r_{t}=\left\{1 \leq j \leq e \mid e_{j} \varepsilon_{t} \neq 0\right\}$ for $1 \leq t \leq a+e$ (then $r_{1}=\ldots=r_{a}$ ).

For $n \in \mathbb{N}$ let

$$
H_{n}=\left(\begin{array}{cccc}
0 & 1 & \ldots & 1 \\
\vdots & \ddots & \ddots & \vdots \\
0 & \ldots & 0 & 1 \\
0 & \ldots & \ldots & 0
\end{array}\right)
$$

be the $n \times n$-matrix having entries above the main diagonal all equal to 1 , and the remaining ones all equal to 0 . Then by [14, Theorem VIII.3],

$$
\varepsilon_{t} \Lambda \cong \begin{cases}\Lambda\left(R_{t}, 1, \ldots, 1, H_{\left|r_{t}\right|}\right) & (1 \leq t \leq a) \\ \Lambda\left(R_{t}, 1, \ldots, 1, a H_{\left|r_{t}\right|}\right) & (a+1 \leq t \leq a+e) .\end{cases}
$$

To describe the exceptional vertex $\Gamma_{a}$, let $\Gamma_{t}=\left(\varepsilon_{1}+\ldots+\varepsilon_{t}\right) \Lambda$ for $1 \leq t \leq$ $a$. Then $\Gamma_{t}$ is an amalgam of $\Gamma_{t-1}$ and $\varepsilon_{t} \Lambda$. More precisely, let

$$
X_{t}=\varepsilon_{t} \Lambda / \operatorname{rad}\left(\varepsilon_{t} \Lambda\right)^{x_{t}}=\varepsilon_{t} \Lambda / \pi_{t}^{x_{t} /\left|r_{1}\right|} \varepsilon_{t} \Lambda \quad \text { where } \quad x_{t}=\left|r_{1}\right| \frac{p^{t-1}-1}{e} .
$$

Then by [14, Theorem VIII.5], there are epimorphisms $v_{t}: \varepsilon_{t} \Lambda \rightarrow X_{t}$ and $\varphi_{t}: \Gamma_{t-1} \rightarrow X_{t}$ such that $\Gamma_{t}=\left\{(\gamma, \lambda) \in \Gamma_{t-1} \oplus \varepsilon_{t} \Lambda \mid \varphi_{t}(\gamma)=v_{t}(\lambda)\right\}$.

LEMMA 3.2. Outcent $0\left(\Gamma_{a}\right)=1$.

Proof. We prove by induction on $t$ that Outcent ${ }_{0}\left(\Gamma_{t}\right)=1$ for $1 \leq t \leq a$. For $t=1$, this follows from Lemma 2.1, so let $t>1$ and $\alpha \in$ Autcent $_{0}\left(\Gamma_{t}\right)$. Then $\alpha$ induces automorphisms $\alpha_{1} \in \operatorname{Autcent}_{0}\left(\Gamma_{t-1}\right)$ and $\alpha_{2} \in \operatorname{Autcent}_{0}\left(\varepsilon_{t} \Lambda\right)$, which on their part induce equal automorphisms of $X_{t}$. By the induction hypothesis, both $\alpha_{1}$ and $\alpha_{2}$ are inner automorphisms, say conjugation with $\gamma \in$ $\Gamma_{t-1}^{\times}$and $\lambda \in\left(\varepsilon_{t} \Lambda\right)^{\times}$, respectively. Since $\varphi_{t}$ and $v_{t}$ are surjective, it follows that $\varphi_{t}(\gamma) v_{t}(\lambda)^{-1}$ is central in $X_{t}$. From the description of $X_{t}$ it follows that $\mathrm{Z}\left(X_{t}\right)=R_{t} / \pi_{t}^{x_{t} /\left|r_{1}\right|} R_{t}$, so we can choose $r \in R_{t}^{\times}$such that $\varphi_{t}(\gamma)=v_{t}(r \lambda)$. This implies that $\alpha$ is an inner automorphism, given by conjugation with $(\gamma, r \lambda) \in \Gamma_{t}^{\times}$. 
If one omits the exceptional vertex from the Brauer tree, one gets a union of trees, each having a vertex with an additional free edge (the one connecting the tree to the exceptional vertex). Let $T$ be such a tree with $t$ vertices, corresponding to $\varepsilon_{i_{1}}, \ldots, \varepsilon_{i_{t}}$. Set $\Delta_{T}=\Lambda\left(\varepsilon_{i_{1}}+\ldots+\varepsilon_{i_{t}}\right)$ and denote by $e_{T} \in \Delta_{T}$ a lift of the central primitive idempotent of $\Delta_{T} / \operatorname{rad}\left(\Delta_{T}\right)$ that corresponds to the free edge of $T$. Note that $e_{T} \Delta_{T} e_{T} \cong R$. Thus it follows from Corollary 2.3 that:

LEMMA 3.3. Every $\alpha \in$ Autcent $_{0}\left(\Delta_{T}\right)$ is conjugation with a unit $A \in \Delta_{T}^{\times}$, and if $x \in\left(e_{T} \Delta_{T} e_{T}\right)^{\times}$, one can assume in addition that $e_{T} A e_{T}=x$.

Set $\Gamma_{0}=\left(\varepsilon_{a+1}+\ldots+\varepsilon_{a+e}\right) \Lambda$. By [14, Theorem VIII.5 (iii)], there are epimorphisms $\mu$ and $v$ of $\Gamma_{a}$ and $\Gamma_{0}$ onto $\bigoplus_{i \in r_{1}} R / p^{a} R$ such that $\Lambda=\{(x, y) \in$ $\left.\Gamma_{a} \oplus \Gamma_{0} \mid \mu(x)=v(y)\right\}$.

Now we can finish the proof of Theorem 3.1. Let $\alpha \in$ Autcent $_{0}(\Lambda)$; we have to show that $\alpha \in \operatorname{Inn}(\Lambda)$. The automorphism $\alpha$ induces automorphisms $\alpha_{1} \in$ Autcent $_{0}\left(\Gamma_{a}\right)$ and $\alpha_{2} \in$ Autcent $_{0}\left(\Gamma_{0}\right)$. By Lemma 3.2, $\alpha_{1}$ is conjugation with some $A_{1} \in \Gamma_{a}^{\times}$. By Lemma 3.3, there is $A_{2} \in \Gamma_{0}^{\times}$with $v\left(A_{2}\right)=\mu\left(A_{1}\right)$ such that $\alpha_{2}$ is conjugation with $A_{2}$. Hence $\alpha$ is conjugation with $\left(A_{1}, A_{2}\right) \in \Lambda^{\times}$.

\section{The principal block of $\mathbb{Z}_{3} A_{6}$}

Let $\mathrm{B}_{0}^{(3)}\left(A_{6}\right)$ be the principal block of $\mathbb{Z}_{3} A_{6}$. Since $A_{6}=\mathrm{PSL}_{2}(9)$ and $\mathrm{SL}_{2}(9)$ have the same principal 3-block, we may apply results from [13] to show that:

THEOREM 4.1. Outcent $\left(\mathrm{B}_{0}^{(3)}\left(A_{6}\right)\right)=1$.

Proof. Let $R=\mathbb{Z}_{3}[\sqrt{5}]$, with field of fractions $K$, and let $\Lambda$ be the principal block of $R A_{6}$. Note that the principal block idempotent of $\mathbb{Z}_{3} A_{6}$ remains primitive in $R A_{6}$. Thus it suffices, by the remark at the end of Section 1, to show that Outcent $(\Lambda)=1$. By Proposition 1.7, this is equivalent to Outcent ${ }_{0}(O)=$ 1 , where $O$ is the basic $R$-order that is Morita equivalent to $\Lambda$.

The ordinary irreducible characters in $\Lambda$ are $1,5 a, 5 b, 8 a, 8 b$ and 10 . We use them to label the central primitive idempotents of $K O$ as $\varepsilon_{1}, \varepsilon_{5 a}, \varepsilon_{5 b}, \varepsilon_{8 a}$, $\varepsilon_{8 b}$ and $\varepsilon_{10}$. The irreducible Brauer characters in $\Lambda$ are $1,4,3 a$ and $3 b$, and they are used to label lifts of the central primitive idempotents in $O / \operatorname{rad}(O)$ (which are orthogonal and sum up to 1 ) as $e_{1}, e_{4}, e_{3 a}$ and $e_{3 b}$. From [13] we know that

$$
\begin{aligned}
& O \varepsilon_{1}=\Lambda(R, 1,(0)) \\
& O \varepsilon_{5 a}=\Lambda\left(R, 1,4,\left(\begin{array}{ll}
0 & 1 \\
0 & 0
\end{array}\right)\right), O \varepsilon_{5 b}=\Lambda\left(R, 1,4,\left(\begin{array}{ll}
0 & 1 \\
0 & 0
\end{array}\right)\right), \\
& O \varepsilon_{8 a}=\Lambda\left(R, 1,4,3 a,\left(\begin{array}{lll}
0 & 1 & 2 \\
0 & 0 & 1 \\
0 & 0 & 0
\end{array}\right)\right), O \varepsilon_{8 b}=\Lambda\left(R, 1,4,3 b,\left(\begin{array}{lll}
0 & 1 & 2 \\
0 & 0 & 1 \\
0 & 0 & 0
\end{array}\right)\right),
\end{aligned}
$$




$$
O \varepsilon_{10}=\Lambda\left(R, 4,3 a, 3 b,\left(\begin{array}{ccc}
0 & 1 & 1 \\
0 & 0 & 1 \\
0 & 1 & 0
\end{array}\right)\right) .
$$

(The Brauer characters are used to indicate which matrix entry belongs to which $e_{i}$.)

Let $\alpha \in$ Autcent $_{0}(O)$; we have to show that $\alpha \in \operatorname{Inn}(O)$. We may assume that $\alpha$ fixes all $e_{i}$. Then $\alpha$ is conjugation with $A \in K O$, a sum of diagonal matrices by Corollary 2.2. Multiplying $A$ by scalar multiples of the central primitive idempotents $\varepsilon_{t}$ in each component, we may assume that $A$ is of the form

$$
\left(1, \operatorname{diag}\left(1, a_{1}\right), \operatorname{diag}\left(1, a_{2}\right), \operatorname{diag}\left(1, a_{3}, b_{1}\right), \operatorname{diag}\left(1, a_{4}, c_{1}\right), \operatorname{diag}\left(1, b_{2}, c_{2}\right)\right)
$$

with $a_{1}, \ldots, c_{2} \in R^{\times}$.

By [13], the bimodule $e_{4} O e_{1}$ is generated by the lines of the following matrix

\begin{tabular}{cccr}
$5 a$ & $5 b$ & $8 a$ & $8 b$ \\
\hline 1 & 0 & 1 & 1 \\
0 & 1 & 1 & -1 \\
0 & 0 & 3 & 0 \\
0 & 0 & 0 & 3
\end{tabular}

Note that $\alpha$ maps $e_{4} O e_{1}$ onto itself; the first two generators are mapped to $\left(a_{1}, 0, a_{3}, a_{4}\right)$ and $\left(0, a_{2}, a_{3},-a_{4}\right)$, respectively. Since $e_{4} O e_{1}$ is indecomposable as bimodule, $a_{1} \equiv a_{2} \equiv a_{3} \equiv a_{4}(\bmod 3)$. Also $e_{4} O e_{3 a}$ and $e_{4} O e_{3 b}$ are indecomposable bimodules, so that $a_{3}^{-1} b_{1} \equiv b_{2}(\bmod 3)$ and $a_{4}^{-1} c_{1} \equiv c_{2}$ $(\bmod 3)$.

If $a, b, c \in R^{\times}$, then $e_{1}+a e_{4}+b e_{3 a}+c e_{3 b}$ is a unit in $O^{\times}$. Modifying $\alpha$ with an inner automorphism which is conjugation with such a unit (if necessary), we may assume that all $a_{i}, b_{i}$ and $c_{i}$ are congruent to 1 modulo 3 . (On the other hand, any such matrix $A$ preserves $O$ since $3 e_{i} \varepsilon_{t} O e_{j} \subset e_{i} O e_{j}$ for $i \neq j$ and all $t$.)

By the description of the orders $e_{i} O e_{i}$ in [13], $3 e_{i} \varepsilon_{t} \pm 3 e_{i} \varepsilon_{10} \in O$ for all $i=4,3 a, 3 b$ and all $t$ with $e_{i} \varepsilon_{t} \neq 0$. Therefore there is $B \in O^{\times}$such that

$$
\begin{aligned}
A B= & (1, \operatorname{diag}(1,1), \operatorname{diag}(1,1), \operatorname{diag}(1,1,1), \operatorname{diag}(1,1,1), \\
& \operatorname{diag}(1+3 u, 1+3 v, 1+3 w))
\end{aligned}
$$

for some $u, v, w \in R$. Multiplying $A B$ with a central unit in $K O$ we obtain

$$
\begin{aligned}
A_{x, y}= & (1, \operatorname{diag}(1,1), \operatorname{diag}(1,1), \operatorname{diag}(1,1,1), \operatorname{diag}(1,1,1), \\
& \operatorname{diag}(1,1+3 x, 1+3 y))
\end{aligned}
$$


with $x, y \in R$, and $\alpha$ differs from conjugation with $A_{x, y}$ by an inner automorphism of $O$ (conjugation with $B$ ). The element

$$
\begin{aligned}
& (1, \operatorname{diag}(1-3 x, 1-3 x), \operatorname{diag}(1-3 y, 1-3 y), \operatorname{diag}(1+3 x, 1+3 x, 1+3 x), \\
& \operatorname{diag}(1+3 y, 1+3 y, 1+3 y), \operatorname{diag}(1,1+3 x, 1+3 y))
\end{aligned}
$$

lies in $O^{\times}$and differs from $A_{x, y}$ by a central unit in $K O$. Thus $\alpha \in \operatorname{Inn}(O)$, and the theorem is proved.

COROLLARY 4.2. For $A_{6}$, a '3-adic version' of the conjecture of Zassenhaus holds: $\operatorname{Aut}\left(\mathbb{Z}_{3} A_{6}\right)=\operatorname{Aut}\left(A_{6}\right) \operatorname{Inn}\left(\mathbb{Z}_{3} A_{6}\right)$.

Proof. This follows from Theorem 4.1, since Out $\left(A_{6}\right)$ is the automorphism group of the character table of $A_{6}$, and $\mathbb{Z}_{3} A_{6}$ has only two blocks: the principal block and a block of defect zero.

REMARK 4.3. Let $k$ be a field of odd characteristic $p>0$, and $G$ a finite group. There are embeddings

$$
\operatorname{Outcent}\left(\mathbb{Z}_{p} G\right) \hookrightarrow \operatorname{Outcent}\left(\mathbb{F}_{p} G\right) \hookrightarrow \operatorname{Outcent}(k G) \text {, }
$$

the first one by Theorem 1.8, the second by the Noether-Deuring theorem. Combined with the inclusion Outcent $(k G) \hookrightarrow \operatorname{Picent}(k G) \cong \operatorname{Picent}(\Lambda)$, where $\Lambda$ is the basic order of $k G$, we obtain an inclusion Outcent $\left(\mathbb{Z}_{p} G\right) \hookrightarrow \operatorname{Picent}(\Lambda)$ (the image consisting of classes of bimodules which are free from one side). In particular, Outcent $(\Lambda)=1$ would imply that $\operatorname{Outcent}\left(\mathbb{Z}_{p} G\right)=1$, and similar remarks are valid for blocks of $\mathbb{Z}_{p} G$. However, the inclusion is usually strict, as the following theorem shows.

THEOREM 4.4. Let $k$ be a field of characteristic 3 which is a splitting field for $\mathbb{F}_{3} A_{6}$ (i.e., $\mathbb{F}_{3^{2}} \subseteq k$ ), and let $\Lambda$ be the basic algebra of the principal block of $k A_{6}$. Then Outcent $(\Lambda)$ is isomorphic to the direct product of the multiplicative group of $k$ and the additive group of $k$.

Proof. By [11], $\Lambda$ is given by its Ext-quiver and relations as shown in Figure 1. It is straightforward to check that $\Lambda$ has $k$-dimension 36. In Figure 2,

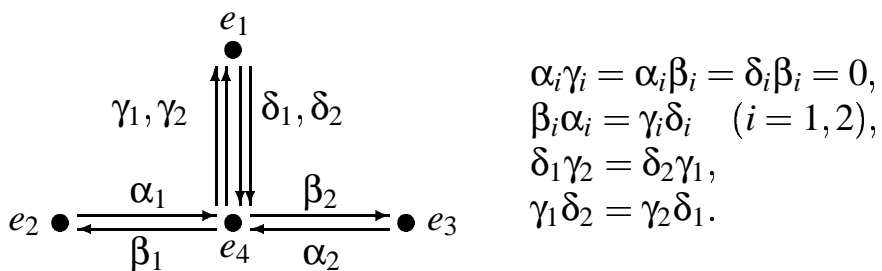

Figure 1. Quiver and relations for $\Lambda$

a $k$-basis of $e_{i} \Lambda e_{j}$ is displayed at $(i, j)$-th position. The dimension of the center 


\begin{tabular}{llll}
\hline $\begin{array}{lll}\left\{e_{1},\right. \\
\delta_{1} \gamma_{1}, \delta_{1} \gamma_{2}, \delta_{2} \gamma_{2},\end{array}$ & $\left\{\delta_{2} \beta_{1}\right\}$ & $\left\{\delta_{1} \beta_{2}\right\}$ & \\
$\left.\delta_{1} \gamma_{1} \delta_{2} \gamma_{2}\right\}$ & & & \\
& & & \\
& & & \\
& & & \\
& & \\
& & $\left.\delta_{1} \delta_{2}, \delta_{2} \gamma_{2} \delta_{1}\right\}$ \\
$\left\{\alpha_{2}\right.$, & & $\left\{\alpha_{1}\right.$, \\
& $\left.\alpha_{1} \gamma_{2} \delta_{2} \beta_{1}\right\}$ & $\left\{\alpha_{1} \beta_{2}\right\}$ & $\left.\alpha_{1} \gamma_{2} \delta_{2}\right\}$ \\
& & & \\
$\left\{\alpha_{2} \gamma_{1}\right\}$ & $\left\{\alpha_{2} \beta_{1}\right\}$ & $\left.\alpha_{2} \gamma_{1} \delta_{1} \beta_{2}\right\}$ & $\left.\alpha_{2} \gamma_{1} \delta_{1}\right\}$ \\
& & & \\
$\left\{\gamma_{1}, \gamma_{2}\right.$, & $\left\{\beta_{1}\right.$, & $\left\{\beta_{2}\right.$, & $\left\{e_{4}\right.$, \\
$\left.\gamma_{1} \delta_{1} \gamma_{2}, \gamma_{2} \delta_{2} \gamma_{1}\right\}$ & $\left.\gamma_{2} \delta_{2} \beta_{1}\right\}$ & $\left.\gamma_{1} \delta_{1} \beta_{2}\right\}$ & $\gamma_{1} \delta_{1}, \gamma_{1} \delta_{2}, \gamma_{2} \delta_{2}$, \\
& & & $\left.\gamma_{1} \delta_{1} \gamma_{2} \delta_{2}\right\}$ \\
\hline
\end{tabular}

Figure 2. Pierce decomposition of $\Lambda$

$\mathrm{Z}(\Lambda)$ of $\Lambda$ is the number of irreducible complex characters belonging to the principal block of $k A_{6}$, and it is easily checked that

$$
\mathrm{Z}(\Lambda)={ }_{k}\left\langle 1, \delta_{1} \gamma_{1} \delta_{2} \gamma_{2}, \alpha_{1} \gamma_{2} \delta_{2} \beta_{1}, \alpha_{2} \gamma_{1} \delta_{1} \beta_{2}, \gamma_{1} \delta_{1} \gamma_{2} \delta_{2}, \delta_{1} \gamma_{2}+\gamma_{1} \delta_{2}\right\rangle .
$$

Let $\Phi \in \operatorname{Autcent}(\Lambda)$. We will seek for a "canonical" representative of the $\operatorname{coset} \Phi \cdot \operatorname{Inn}(\Lambda)$. First of all, we can assume that $\Phi$ stabilizes the set of idempotents $\left\{e_{1}, e_{2}, e_{3}, e_{4}\right\}$. From the description of $\mathrm{Z}(\Lambda)$ we see that $\Phi$ fixes $e_{2}$ and $e_{3}$, and obviously no automorphism of $\Lambda$ interchanges $e_{1}$ and $e_{4}$. Thus $\Phi$ fixes each $e_{i}$.

We still have the freedom to modify $\Phi$ by inner automorphisms given by conjugation with 'diagonal' units $\sum_{i=1}^{4} \lambda_{i}$, where $\lambda_{i} \in\left(e_{i} \Lambda e_{i}\right)^{\times}$. Let $t, v \in k$ and $s, u \in k^{\times}$. With the unit

$$
v=e_{1}+s^{-1} e_{2}+u^{-1} e_{3}+\left(e_{4}+u^{-1} v \gamma_{1} \delta_{1}+s^{-1} t \gamma_{2} \delta_{2}\right) \in \Lambda^{\times}
$$

one calculates $v^{-1} \alpha_{1} v=s \alpha_{1}+t \alpha_{1} \gamma_{2} \delta_{2}$ and $v^{-1} \alpha_{2} v=u \alpha_{2}+v \alpha_{2} \gamma_{1} \delta_{1}$. Thus we may assume that $\Phi$ fixes $\alpha_{1}$ and $\alpha_{2}$. Then the relations $\Phi\left(\alpha_{i}\right) \Phi\left(\beta_{i}\right)=0$ show that $\Phi\left(\beta_{1}\right)=x \beta_{1}$ and $\Phi\left(\beta_{2}\right)=y \beta_{2}$ for some $x, y \in k^{\times}$. The relations $\Phi\left(\delta_{i}\right) \Phi\left(\beta_{i}\right)=0$ show that $\Phi\left(\delta_{1}\right)=a \delta_{1}+b \delta_{1} \gamma_{1} \delta_{2}+c \delta_{2} \gamma_{2} \delta_{1}$ and $\Phi\left(\delta_{2}\right)=$ $l \delta_{2}+m \delta_{1} \gamma_{1} \delta_{2}+n \delta_{2} \gamma_{2} \delta_{1}$ with $b, c, m, n \in k$ and $a, l \in k^{\times}$. The unit

$$
\mu=\left(a^{-1} e_{1}-a^{-1} m l^{-1} \delta_{1} \gamma_{1}-b a^{-2} \delta_{1} \gamma_{2}-c a^{-2} \delta_{2} \gamma_{2}\right)+e_{2}+e_{3}+e_{4} \in \Lambda^{\times}
$$

commutes with the $\alpha_{i}$ and $\beta_{i}$. Replacing $\Phi$ by the map $\lambda \mapsto \mu \Phi(\lambda) \mu^{-1}$ one obtains furthermore $\Phi\left(\delta_{1}\right)=\delta_{1}$ and $\Phi\left(\delta_{2}\right)=s \delta_{2}+t \delta_{2} \gamma_{2} \delta_{1}$ with $s \in k^{\times}$and $t \in$ 
$k$. The condition that $\Phi$ fixes the central element $\delta_{1} \gamma_{2}+\gamma_{2} \delta_{1}$ yields $\Phi\left(\gamma_{2}\right)=$ $\gamma_{2}+a \gamma_{1} \delta_{1} \gamma_{2}$ for some $a \in k$. The relation $\Phi\left(\gamma_{2}\right) \Phi\left(\delta_{2}\right)=\Phi\left(\beta_{2} \alpha_{2}\right)=y \beta_{2} \alpha_{2}$ forces $s=y$ and $a=0$. From $\Phi\left(\gamma_{1}\right) \delta_{1}=\Phi\left(\gamma_{1} \delta_{1}\right)=x \beta_{1} \alpha_{1}$ it follows that $\Phi\left(\gamma_{1}\right)=x \gamma_{1}+x c \gamma_{1} \delta_{1} \gamma_{2}$ for some $c \in k$. Finally, $\Phi\left(\delta_{2}\right) \Phi\left(\gamma_{1}\right)=\Phi\left(\delta_{1} \gamma_{2}\right)=\delta_{1} \gamma_{2}$ yields $y=x^{-1}$ and $y x c+x t=0$. Thus

$$
\begin{aligned}
& \Phi\left(\alpha_{1}\right)=\alpha_{1}, \Phi\left(\beta_{1}\right)=x \beta_{1}, \quad \Phi\left(\delta_{1}\right)=\delta_{1}, \Phi\left(\gamma_{1}\right)=x\left(\gamma_{1}+c \gamma_{1} \delta_{1} \gamma_{2}\right), \\
& \Phi\left(\alpha_{2}\right)=\alpha_{2}, \Phi\left(\beta_{2}\right)=x^{-1} \beta_{2}, \Phi\left(\gamma_{2}\right)=\gamma_{2}, \Phi\left(\delta_{2}\right)=x^{-1}\left(\delta_{2}-c \delta_{2} \gamma_{2} \delta_{1}\right) .
\end{aligned}
$$

Conversely, for each $x \in k^{\times}$and $c \in k$, this defines a central automorphism $\Phi=\Phi_{x, c}$ of $\Lambda$. From $\Phi_{x, c} \Phi_{x^{\prime}, c^{\prime}}=\Phi_{x x^{\prime}, c+c^{\prime}}$ it follows that these automorphisms generate a group isomorphic to the direct product of the multiplicative group of $k$ and the additive group of $k$. It is easily verified that each $\Phi_{x, c}$ is a noninner automorphism of $\Lambda$. (For example by showing that the existence of $\lambda_{1} \in\left(e_{1} \Lambda e_{1}\right)^{\times}$and $\lambda_{4} \in\left(e_{4} \Lambda e_{4}\right)^{\times}$with $\lambda_{1} \delta_{i} \lambda_{4}=\Phi_{x, c}\left(\delta_{i}\right)$ for $i=1,2$ implies that $x=1$ and $c=0$.)

Let $B_{0}$ be the principal block of a $p$-adic group ring, with defect group $P$. It seems to be an interesting question whether central automorphisms of $B_{0}$ which stabilize $P$ are necessarily inner automorphisms. Here, we prove this for the principal $p$-blocks of the groups $\mathrm{SL}_{2}\left(p^{f}\right)(p$ a prime, $f \in \mathbb{N}$ ). The proof splits into two parts: in the next lemma we record a simple group theoretical fact about these groups, and then the proof is completed using Green correspondence of automorphisms.

LEMMA 4.5. Set $G=\mathrm{PSL}_{2}\left(p^{f}\right)$, and let $H$ be the normalizer in $G$ of a Sylow $p$-subgroup $P$ of $G$. Let $\sigma \in \operatorname{Aut}(H)$ be such that $x$ and $\sigma(x)$ are conjugate in $G$ for all $x \in P$. Then $\sigma$ can be extended to an automorphism of $G$.

Proof. Note that $H$ is the image of a Borel subgroup of $\mathrm{SL}_{2}\left(p^{f}\right)$ under the natural map $\mathrm{SL}_{2}\left(p^{f}\right) \rightarrow G$. Let $F$ be the field with $p^{f}$ elements, and $\omega$ a generator of $F^{\times}$. Since $\left[\begin{array}{cc}d & 0 \\ 0 & d^{-1}\end{array}\right]\left[\begin{array}{cc}1 & a \\ 0 & 1\end{array}\right]\left[\begin{array}{cc}d^{-1} & 0 \\ 0 & d\end{array}\right]=\left[\begin{array}{cc}1 & a d^{2} \\ 0 & 1\end{array}\right]$ for all $a, d \in F^{\times}$, we may identify $H$ with a semidirect product $F \rtimes K$, where $K$ acts via an isomorphism $\mu: K \rightarrow\left\langle\omega^{2}\right\rangle$ on the additive group $F$ by multiplication. Modifying $\sigma$ by an inner automorphism (which clearly extends to an automorphism of $G$ ) we may assume that $\sigma$ stabilizes $K$. Then $\sigma$ induces automorphisms $\alpha \in \operatorname{Aut}(F)$ and $\beta \in \operatorname{Aut}(K)$. Note that we have identified $P$ with $F$, and that $p$-elements of $G$ are conjugate in $G$ if their preimages in $\mathrm{SL}_{2}\left(p^{f}\right)$ are conjugate. It follows that for $x \in F$, the elements $x$ and $\alpha(x)$ are conjugate in $H$, and we may assume additionally that $\alpha(1)=1$. Since $\sigma$ is a homomorphism,

$$
\alpha(x \cdot \mu(k))=\alpha(x) \cdot \mu(\beta(k)) \quad \text { for all } x \in F \text { and } k \in K .
$$

Choosing $x=1$ gives $\mu(\beta(k))=\alpha(\mu(k))$, so $(* *) \alpha(x s)=\alpha(x) \alpha(s)$ for all $x \in F$ and $s \in\left\langle\omega^{2}\right\rangle$. Let $x, y \in F$. Since each element of $F$ is the sum of two 
squares, $y=a^{2}+b^{2}$ for some $a, b \in F$, and

$$
\begin{aligned}
\alpha(x y) & =\alpha\left(x a^{2}+x b^{2}\right)=\alpha\left(x a^{2}\right)+\alpha\left(x b^{2}\right) \\
& \stackrel{(* *)}{=} \alpha(x) \alpha\left(a^{2}\right)+\alpha(x) \alpha\left(b^{2}\right)=\alpha(x) \alpha\left(a^{2}+b^{2}\right)=\alpha(x) \alpha(y) .
\end{aligned}
$$

Thus $\alpha$ is in fact a field automorphism of $F$. Modifying $\sigma$ by an automorphism induced by a field automorphism of $G$, we may assume that $\alpha$ is the identity mapping. But then $(*)$ implies that $\beta$ is also the identity mapping, and the lemma is proved.

Recall that for a block $B$ with defect group $D$ we denote by $\operatorname{Aut}_{D}(B)$ the group of automorphisms of $B$ which stabilize $D$.

PROPOSITION 4.6. Let $B_{0}$ be the principal p-block of $S L_{2}\left(p^{f}\right)$, with defect group $P$. Then

$$
\operatorname{Outcent}\left(B_{0}\right) \cap \operatorname{Out}_{P}\left(B_{0}\right)=1 \text {. }
$$

Proof. The block $B_{0}$ is the principal $p$-block of $G=\operatorname{PSL}_{2}\left(p^{f}\right)$. Let $P$ be a Sylow $p$-subgroup of $G$, set $H=\mathrm{N}_{G}(P)$ and let $b_{0}$ be the Brauer correspondent for $H$ to $B_{0}$ (note that $b_{0}=R H$ ). We assume that $P \subset b_{0} \subset B_{0}$.

Let $\alpha \in \operatorname{Autcent}\left(B_{0}\right)$ with $\alpha(P)=P$; we have to show that $\alpha \in \operatorname{Inn}\left(B_{0}\right)$. By the Green correspondence for automorphisms ([15, (4.8)]) which we already mentioned in the introduction, the Green correspondent of $\alpha_{\alpha}\left(B_{0}\right)_{1}$ is $\beta\left(b_{0}\right)_{1}$ for some $\beta \in \operatorname{Aut}\left(b_{0}\right)$ with $\left.\alpha\right|_{P}=\left.\beta\right|_{P}$; moreover $\beta \in \operatorname{Aut}_{\mathrm{n}}\left(b_{0}\right)$ since $\alpha \in \operatorname{Aut}_{\mathrm{n}}\left(B_{0}\right)$. In particular $\beta(P)=P$, and there is $\sigma \in \operatorname{Aut}(H)$ with $\left.\sigma\right|_{P}=\left.\beta\right|_{P}$ and $\sigma^{-1} \beta \in \operatorname{Inn}\left(b_{0}\right)$ (see [9]). Let $x \in P$. As central automorphism, $\alpha$ fixes all irreducible characters $\chi$ belonging to $B_{0}$. Therefore $\sum_{\chi \in B_{0}} \chi(\alpha(x)) \chi\left(x^{-1}\right)=$ $\sum_{\chi \in B_{0}}|\chi(x)|^{2} \neq 0$, and it follows from block orthogonality that $x$ and $\alpha(x)$ are conjugate in $G$. Hence Lemma 4.5 applies to give $\hat{\sigma} \in \operatorname{Aut}(G)$ with $\left.\hat{\sigma}\right|_{H}=\sigma$. The Green correspondent to $\hat{\sigma}^{-1} \alpha\left(B_{0}\right)_{1}$ is clearly $\sigma^{-1} \beta\left(b_{0}\right)_{1}$, which is isomorphic to the 'untwisted' bimodule ${ }_{1}\left(b_{0}\right)_{1}$. Thus ${ }_{\hat{\sigma}^{-1} \alpha}\left(B_{0}\right)_{1}$ and ${ }_{1}\left(B_{0}\right)_{1}$ have the same Green correspondent, and therefore $\alpha \in \hat{\sigma} \cdot \operatorname{Inn}\left(B_{0}\right)$. It follows that $\hat{\sigma}$ fixes all irreducible characters belonging to $B_{0}$. Since there is only one other block, which is of defect zero, $\hat{\sigma}$ fixes all irreducible characters of $G$ (equivalently, all conjugacy classes of $G$ ), and it follows easily that $\hat{\sigma} \in \operatorname{Inn}(G)$, so $\alpha \in \operatorname{Inn}\left(B_{0}\right)$, as desired. 


\section{An application using the small group ring}

In this section, we verify the Zassenhaus conjecture $(\mathrm{ZC})_{\text {Aut }}$ for the perfect group $G$ of order 1080. This group is a central extension of the group $A_{6}$ by a cyclic group of order 3 , and is denoted by $3 . A_{6}$.

We briefly explain where our interest in this task comes from. The automorphisms of a finite Coxeter group $W$, its integral group ring $\mathbb{Z} W$, and the associated generic Iwahori-Hecke algebra are classified in [2] (in particular, $(\mathrm{ZC})_{\text {Aut }}$ holds for $W$ ), and in [2, p. 620] the opinion has been expressed that at least some of these results should extend to the case where $W$ is a finite complex reflection group. Shephard and Todd classified in [20] the finite complex reflection groups. These groups are direct products of irreducible ones, which either belong to one of three infinite series or to a list of 34 groups. A complete list of these groups together with presentations, symbolized by diagrams "à la Coxeter", is given in [5]. For all except one group from the list of 34 groups, Greiner showed in her diploma thesis [8] that (ZC) Aut is valid. This was done by applying the $\mathrm{F}^{*}$-Theorem (mentioned in the introduction) and the methods from [3]. The remaining group is number 27 from [20, Table VII], denoted by $G_{27}$ in [5]. It is the direct product of a cyclic group of order 2 and the perfect group 3. $A_{6}$. It is known that if $G$ and $H$ are finite groups which are determined by their integral group rings, $(\mathrm{ZC})_{\text {Aut }}$ holds for the direct product $G \times H$ provided (ZC) Aut holds for the factors $G$ and $H$ (see the discussion in $\left[3\right.$, Remark 3.3]). Thus, in order to show that (ZC) Aut holds for $G_{27}$, it suffices to verify the conjecture for $3 . A_{6}$. We remark that application of currently known methods (see [3]) does not yield this result. Instead, we first review some aspects of the "method of the small group ring". Then we shall see that Theorem 4.1 implies the desired result.

Let $G$ be a finite group, and $R$ a commutative ring. For a subgroup $U$ of $G$, we write $\mathrm{I}_{R}(U)$ for the augmentation ideal of $R U$ (the elements of $R U$ of augmentation 0). If $U \unlhd G$, then the two-sided ideal $\mathrm{I}_{R}(U) G$ of $R G$ generated by $\mathrm{I}_{R}(U)$ is the kernel of the natural map $R G \rightarrow R G / U$. If $R=\mathbb{Z}$, we omit the subscript $R$.

Let $A$ be a normal abelian subgroup of $G$, and consider the exact sequence

$$
0 \longrightarrow \frac{\mathrm{I}(A) G}{\mathrm{I}(A) \mathrm{I}(G)} \longrightarrow \frac{\mathbb{Z} G}{\mathrm{I}(A) \mathrm{I}(G)} \longrightarrow \mathbb{Z} G / A \longrightarrow 0
$$

The middle term is called the small group ring of $G$ over $\mathbb{Z}$ associated with $G$ and $A$ (see $[16,1.1 .8]$ ), and will be denoted by

$$
s(G, A) .
$$

It is well known that the kernel of the natural surjection $s(G, A) \rightarrow \mathbb{Z} G / A$ is isomorphic to $A$ as left $\mathbb{Z} G$-module, the action of $G$ on $A$ given by conjugation. 
An isomorphism is obtained by sending $a$ in $A$ to the coset of $a-1$, and we suggestively write $\{A-1\}$ for the kernel. Note that the right action of $G$ on $\{A-1\}$ is trivial. Tensoring the exact sequence with an integral domain $R$ of characteristic 0 over $\mathbb{Z}$ yields the exact sequence

$$
0 \longrightarrow R \otimes_{\mathbb{Z}}\{A-1\} \longrightarrow R \otimes_{\mathbb{Z}} s(G, A) \longrightarrow R G / A \longrightarrow 0 .
$$

We shall write $s_{R}(G, A)=R \otimes_{\mathbb{Z}} s(G, A)=R G / \mathrm{I}_{R}(A) \mathrm{I}_{R}(G)$. Note that $R \otimes_{\mathbb{Z}}$ $\{A-1\}$ is an ideal of square zero, so we get an induced exact sequence of unit groups:

$$
1 \longrightarrow 1+R \otimes_{\mathbb{Z}}\{A-1\} \longrightarrow\left(R \otimes_{\mathbb{Z}} s(G, A)\right)^{\times} \longrightarrow(R G / A)^{\times} \longrightarrow 1 .
$$

Now assume that $A$ is an abelian $p$-group. Taking for $R$ the $p$-adic integers $\mathbb{Z}_{p}$, we obtain the exact sequence

$$
0 \longrightarrow\{A-1\} \longrightarrow s_{\mathbb{Z}_{p}}(G, A) \longrightarrow \mathbb{Z}_{p} G / A \longrightarrow 0
$$

and the induced exact sequence of unit groups

$$
1 \longrightarrow A \longrightarrow\left(s_{\mathbb{Z}_{p}}(G, A)\right)^{\times} \longrightarrow\left(\mathbb{Z}_{p} G / A\right)^{\times} \longrightarrow 1 .
$$

This sequence might be refined as follows. Let $1=\varepsilon_{1}+\ldots+\varepsilon_{n}$ be an orthogonal decomposition of 1 in $\mathbb{Z}_{p} G / A$ into idempotents. (The notation for idempotents should not be confused with the one used in previous sections.) The decomposition can be lifted to an orthogonal decomposition $1=e_{1}+$ $\ldots+e_{n}$ of 1 in $\mathbb{Z}_{p} G$ (with $e_{i}$ mapping to $\varepsilon_{i}$ ), and such a lift is unique up to conjugation by a unit in $\mathbb{Z}_{p} G$ (see [21, §3]). Let $\bar{e}_{i}$ be the image of $e_{i}$ in $s_{\mathbb{Z}_{p}}(G, A)$. As a sequence of additive groups, the above sequence is the direct sum of the exact sequences

$$
0 \longrightarrow \bar{e}_{i}\{A-1\} \bar{e}_{j} \longrightarrow \bar{e}_{i}\left(s_{\mathbb{Z}_{p}}(G, A)\right) \bar{e}_{j} \longrightarrow \varepsilon_{i}\left(\mathbb{Z}_{p} G / A\right) \varepsilon_{j} \longrightarrow 0 .
$$

Now let $\varepsilon$ be the principal block idempotent of $\mathbb{Z}_{p} G / A$, let $e$ be an idempotent of $\mathbb{Z}_{p} G$ which lifts $\varepsilon$, and denote its image in $s_{\mathbb{Z}_{p}}(G, A)$ by $\bar{e}$. Assume that the $G / A$-module $A$ belongs to the principal block $\varepsilon\left(\mathbb{Z}_{p} G / A\right)$. Then $e$ acts as identity on $A$, and since $e$ has augmentation 1, it follows that $\bar{e}\{A-1\} \bar{e}=$ $\{A-1\}$. Thus we obtain the exact sequence

$$
0 \longrightarrow\{A-1\} \longrightarrow \bar{e}\left(s_{\mathbb{Z}_{p}}(G, A)\right) \bar{e} \longrightarrow \varepsilon\left(\mathbb{Z}_{p} G / A\right) \varepsilon \longrightarrow 0,
$$

which gives rise to an exact sequence of unit groups

$$
1 \longrightarrow A \longrightarrow\left(\bar{e}\left(s_{\mathbb{Z}_{p}}(G, A)\right) \bar{e}\right)^{\times} \longrightarrow\left(\varepsilon\left(\mathbb{Z}_{p} G / A\right) \varepsilon\right)^{\times} \longrightarrow 1 .
$$

We remark that $\bar{e}$ is uniquely determined. Indeed, assume that for some $a \in A$, the image of $e+a-1$ in $s_{\mathbb{Z}_{p}}(G, A)$ is an idempotent. Calculating modulo 
$\mathrm{I}_{\mathbb{Z}_{p}}(A) \mathrm{I}_{\mathbb{Z}_{p}}(G)$ yields $e+a-1 \equiv(e+a-1)^{2}=e a+a e-e+(a-1)^{2} \equiv e a+$ $(a-1) e \equiv e a+a-1$. Thus $\bar{e}(a-1)=0$ in $s_{\mathbb{Z}_{p}}(G, A)$, forcing $a=1$.

This implies that $\bar{e}$ is central in $s_{\mathbb{Z}_{p}}(G, A)$. In particular, the preimage of $\varepsilon(G / A)$ in $\bar{e}\left(s_{\mathbb{Z}_{p}}(G, A)\right)$ is the homomorphic image $\bar{e} G$ of $G$. Moreover, if $\mathrm{O}_{p^{\prime}}(G / A)=1$, then $\varepsilon(G / A) \cong G / A$ and consequently $\bar{e} G \cong G$.

We next explain how this might be used to derive information about automorphisms of $\mathbb{Z} G$. Let $\alpha$ be a normalized automorphism of $\mathbb{Z} G$ which stabilizes $\mathrm{I}(A) G$. Then $\alpha$ induces automorphisms of $\mathbb{Z} G / A, \mathbb{Z}_{p} G$, the small group ring $s_{\mathbb{Z}_{p}}(G, A)$ and $\mathbb{Z}_{p} G / A$, which, for simplicity, are also denoted by $\alpha$. It follows that $\alpha(\varepsilon)=\varepsilon$ and $\alpha(\bar{e})=\bar{e}$. Note that the map $\mathbb{Z}_{p} G \rightarrow \mathbb{Z}_{p} G / A$ is surjective on units (see [6, (50.7)]). Thus, if $\alpha$ induces an inner automorphism of $\varepsilon\left(\mathbb{Z}_{p} G / A\right.$ ), we may modify $\alpha$ by an inner automorphism of $\mathbb{Z}_{p} G$ (which, of course, need not stabilize $\mathbb{Z} G$ ) such that $\alpha$ induces the identity mapping on $\varepsilon\left(\mathbb{Z}_{p} G / A\right)$. In particular, $\alpha$ induces an automorphism of $\bar{e} G$ which induces the identity mapping on $\varepsilon(G / A)$.

We record some consequences in a special case. Recall that $\mathrm{B}_{0}^{(p)}(G)$ denotes the principal block of $\mathbb{Z}_{p} G$, and recall our Notation 1.3 for class sums and lengths. It is well known that a normalized automorphism of $\mathbb{Z} G$ maps a class sum $\mathcal{C}_{g}$ to a class sum $\mathcal{C}_{h}$ such that $g$ and $h$ have the same order.

LEMMA 5.1. Let $G$ be a finite group whose center $A=\mathrm{Z}(G)$ is a p-group, and assume that $\mathrm{O}_{p^{\prime}}(G)=1$. Assume further that $(\mathrm{ZC})_{\text {Aut }}$ holds for $G / A$, and that Outcent $\left(\mathrm{B}_{0}^{(p)}(G / A)\right)=1$. Let $\alpha$ be a normalized automorphism of $\mathbb{Z} G$. Then there is $\sigma \in \operatorname{Aut}(G)$ such that for $\tilde{\alpha}=\alpha \sigma^{-1}$ the following hold. For all $g \in G$, there is $a \in A$ (depending on $g$ ) such that $\tilde{\alpha}\left(C_{g}\right)=a C_{g}$. Class sums of $p^{\prime}$-elements, and of elements whose class length is not divisible by $p$, are fixed by $\tilde{\alpha}$, and some p-power of $\tilde{\alpha}$ is a central automorphism.

Proof. By a well known result due to G. Higman, $\alpha(A)=A$, so $\alpha$ stabilizes $\mathrm{I}(A) G$ and induces automorphisms of $\mathbb{Z} G / A$ etc. By assumption, $\alpha$ on $\mathbb{Z} G / A$ is the composition of a group automorphism $\tau$ of $G / A$ and a central automorphism. Let $\varepsilon$ be the principal block idempotent of $\mathbb{Z}_{p} G / A$ and $\bar{e}$ its (unique) lift to an idempotent of $s_{\mathbb{Z}_{p}}(G, A)$. Again by assumption, $\alpha$ on $\varepsilon\left(\mathbb{Z}_{p} G / A\right)$ is the composition of $\tau$ and an inner automorphism (which lifts to an inner automorphism of $\left.\bar{e}\left(s_{\mathbb{Z}_{p}}(G, A)\right)\right)$. Thus $\tau$ lifts to an automorphism $\sigma$ of $\bar{e}\left(s_{\mathbb{Z}_{p}}(G, A)\right)$. Since the preimage of $\varepsilon(G / A)$ is $\bar{e} G$, which is isomorphic to $G$ by assumption, $\sigma$ can be identified with an automorphism of $G$. Put $\tilde{\alpha}=\alpha \sigma^{-1}$, and let $g \in G$. Since $\tilde{\alpha}$ induces central automorphisms of $\mathbb{Z} G / A$ and $\bar{e}\left(s_{\mathbb{Z}_{p}}(G, A)\right)$, there is $a \in A$ with $\tilde{\alpha}\left(C_{g}\right)=C_{a g}=a C_{g}$ and $\tilde{\alpha}(a)=a$. It follows that class sums of $p^{\prime}$-elements are fixed by $\tilde{\alpha}$, and that some $p$-power of $\tilde{\alpha}$ is a central automorphism. Assume that the class length $\left|\mathcal{C}_{g}\right|$ of $g$ is not divisible by $p$. Then $\mathcal{C}_{a g}-\mathcal{C}_{g} \equiv(a-1) \mathcal{C}_{g} \equiv\left|\mathcal{C}_{g}\right|(a-1) \bmod \mathrm{I}_{\mathbb{Z}_{p}}(A) \mathrm{I}_{\mathbb{Z}_{p}}(G)$, and since $s_{\mathbb{Z}_{p}}(G, A)$ has only $p$-torsion (as additive group), $a \equiv 1$ and $a=1$. This proves the lemma. 
THEOREM 5.2. The Zassenhaus conjecture (ZC) Aut holds for $3 . A_{6}$.

Proof. We verify the criteria given in [3, Theorem 3.4], that is, that each automorphism of the character table $T$ of $3 . A_{6}$ is induced by a group automorphism. It can be checked (for example, using GAP [7]), that the automorphism group of $T$ has order 8. (The automorphism group of $3 . A_{6}$ acts as Klein's four group on $T$, so there is really something to prove.) In particular, there are no automorphisms of 3-power order, and the theorem follows from Theorem 4.1 and Lemma 5.1.

\section{References}

1. Alperin, J. L.: 1986, Local representation theory. Cambridge: Cambridge University Press.

2. Bleher, F. M., Geck, M., and Kimmerle, W.: 1997, 'Automorphisms of generic IwahoriHecke algebras and integral group rings of finite Coxeter groups'. J. Algebra 197(2), 615-655.

3. Bleher, F. M., Hiss, G., and Kimmerle, W.: 1995, 'Autoequivalences of blocks and a conjecture of Zassenhaus'. J. Pure Appl. Algebra 103, 23-43.

4. Bleher, F. M. and Kimmerle, W.: 2000, 'On the structure of integral group rings of sporadic groups'. LMS J. Comput. Math. 3, 274-306 (electronic).

5. Broué, M., Malle, G., and Rouquier, R.: 1998, 'Complex reftection groups, braid groups, Hecke algebras'. J. Reine Angew. Math. 500, 127-190.

6. Curtis, C. W. and Reiner, I.: 1981, 1987, Methods of Representation Theory Vol. I, II. Wiley-Interscience, New York.

7. GAP: 2000, 'GAP - Groups, Algorithms, and Programming, Version 4.2'. The GAP Group, Aachen, St Andrews. (http://www-gap.dcs.st-and.ac.uk/ gap) .

8. Greiner, A. R.: 1997, 'Ganzzahlige Gruppenringe komplexer Spiegelungsgruppen'. Master's thesis, Universität Stuttgart.

9. Hertweck, M., 'Automorphisms of principal blocks stabilizing Sylow subgroups'. to appear in Arch. Math. (Basel).

10. Hertweck, M. and Kimmerle, W., 'On principal blocks of p-constrained groups'. to appear in Proc. London Math. Soc. (3).

11. Koshita, H.: 1998, 'Quiver and relations for $\operatorname{SL}\left(2, p^{n}\right)$ in characteristic $p$ with $p$ odd'. Comm. Algebra 26(3), 681-712.

12. Linckelmann, M.: 1996, 'The isomorphism problem for cyclic blocks and their source algebras'. Invent. Math. 125(2), 265-283.

13. Nebe, G.: 1998, 'The group ring of $\mathrm{SL}_{2}\left(p^{2}\right)$ over the $p$-adic integers'. J. Algebra 210(2), 593-613.

14. Plesken, W.: 1983, Group rings of fi nite groups over p-adic integers, Vol. 1026 of Lecture Notes in Math. Berlin: Springer-Verlag.

15. Roggenkamp, K. W.: 1991, The isomorphism problem for integral group rings of fi nite groups, Vol. 95, pp. 193-220. Birkhäuser Verlag Basel.

16. Roggenkamp, K. W. and Scott, L. L.: 1987, 'Isomorphisms of p-adic group rings'. Ann. of Math. 126, 593-647.

17. Scott, L. L.: 1987, 'Recent Progress on the Isomorphism Problem'. Representations of fi nite groups, Proc. Conf., Arcata/Calif. 1986, Proc. Symp. Pure Math. 47(1), 259-274. 
18. Scott, L. L.: 1990, 'Defect groups and the isomorphism problem'. Proc. Colloq. Luminy, France 1988, Astèrisque 181-182 pp. 257-262.

19. Sehgal, S. K.: 1993, Units in integral group rings. Harlow: Longman Scientific \& Technical. With an appendix by Al Weiss.

20. Shephard, G. C. and Todd, J. A.: 1954, 'Finite unitary refection groups'. Canadian J. Math. 6, 274-304.

21. Thévenaz, J.: 1995, G-algebras and modular representation theory, Oxford Mathematical Monographs. Oxford Science Publications. The Clarendon Press, Oxford University Press, New York. 
mit-gabi-v2.tex; 11/10/2001; p.24 